\title{
Association of CD69 and CD 25 Activation Markers on CD4 and CD8 Cells with Skin Tests in Drug Allergy
}

Teixeira FM', Vasconcelos LMF$^{2}$, Araújo TS $^{3}$, Genre $J^{4}$, Almeida TLP ${ }^{5}$, Magalhães HIF ${ }^{3}$, Câmara LMC ${ }^{6}$ and Nagao-Dias AT ${ }^{*}$

${ }^{1}$ Posgraduation Program of Biotechnology (RENORBIO), Federal University of Ceará (UFC), Brazil

${ }^{2}$ Posgraduation Program of Pharmaceutical Sciences, UFC, Brazil

${ }^{3}$ Department of Clinical Analysis and Toxicology, Faculty of Pharmacy, UFC, Brazil

${ }^{4}$ Department of Clinical Analysis and Toxicology, Faculty of Pharmacy, Federal University of Rio Grande do Norte, Brazil

${ }^{5}$ Department of Dermatology, Hospital Universitário Walter Cantídio, UFC, Brazil

${ }^{6}$ Department of Pathology, Faculty of Medicine, UFC, Brazil

\begin{abstract}
Background: Diagnosis of drug allergy is difficult because few methods have been validated in the literature. In the last few years, identification of T cell activation markers to assess drug allergy has been the focus of several studies.

Objective: The aim of the present work was to search for CD25 and CD69 markers on T CD4+ and T CD8+ cells in drug allergy.

Methods: Fourteen patients with drug hypersensitivity were enrolled in this investigation. Some patients had at least one adverse reaction to one or more suspected drugs, therefore, a total of 16 reactions and 10 drugs were investigated. Prick or patch tests were done according with the time of onset and type of the clinical manifestations. In vitro studies were performed by incubating peripheral blood mononuclear cells from patients and controls with different concentrations of the suspicious drugs for 72 hours. The samples were stained with fluorochrome- labelled monoclonal antibodies against CD69, CD25, CD4 and CD8 molecules and analyzed by flow cytometry.

Results: Statistical differences were found at medium and high drug concentrations for the CD4+CD69+ marker $(p \leq 0.05)$, at the lowest drug concentration for the CD4+CD25+ and CD8+CD69+ markers $(p \leq 0.05)$ and at the highest drug concentration for the CD8+CD25+ marker $(p<0.01)$ when samples from patients were compared to controls. One or both the markers were upregulated in 3 patients who presented positive results in prick test.

Four out of six patients who presented positive patch test showed upregulation of one or both the activation markers. For instance, a patient who suffered from pruritus after thediclofenac and ASA ingestion showed a positive prick test for both drugs and presented upregulation of CD69 on CD4+ cells. Another patient who had presented contact dermatitis to rifamycin showed upregulation of CD69 on CD4+ cells, and CD25 on CD4+ and CD8+ cells.

Conclusion: Our data reinforce the use of CD69 and CD25 on both CD4+ and CD8+ T cells in order to investigate drug allergy.
\end{abstract}

Keywords: Lymphocyte activation markers; Drug allergy; Drug hypersensitivity

Abbreviations: DHR: Drug Hypersensitivity Reactions; PBMC: Peripheral Blood Mononuclear Cells; SI: Stimulation Indexes; CM: Culture Medium; NSAIDS: Non-Steroidal Anti-inflammatory Drugs; DRESS: Drug Rash with Eosinophilia and Systemic Symptoms; CT: Healthy Individuals; PT: Allergic Patients; IDT: Intradermal Tests; TEN: Toxic Epidermal Necrolysis; TNF: Tumor Necrosis Factor; Normalized Mean Fluorescence Intensity (NMFI); Acute Generalized Exanthematous Pustulosis (AGEP); Fixed Drug Eruption (FDE); Stevens-Johnson syndrome (SJS); Maculopapular exanthema (MPE)

\section{Introduction}

Drug hypersensitivity reactions (DHR) account for approximately $15 \%$ of all adverse drug reactions [1], can be life threatening, require or prolong the period of hospitalization and entail changes in drug prescription [2]. DHR represent an important problem because they may cause significant morbidity, mortality and high costs for public health systems [3].

Drug allergy is defined by the World Allergy Organization as an immunologically mediated reaction that occurs after re-exposure to the offending drug, through mechanisms mediated or not by IgE [4] that may provoke recurrence upon re-exposure to the drug [5]. The clinical picture of drug hypersensitivity is very heterogeneous and of variable severity [5]. Although hypersensitivity can affect many organ systems, the most common target is the skin [6].

A conclusive laboratory diagnosis of drug allergy that confirms the clinical symptoms and identifies its causative agent still remains a major challenge in daily clinical practice [7]. Skin tests, such as patch, prick and intradermal tests (IDT) may present low sensitivity in patients with a clear history of DHR [5,7]. Although provocation oral tests are considered to be the gold standard in drug allergy, sometimes they are not well accepted by physicians and patients due to the risk of causing severe reactions $[1,8]$.

*Corresponding author: Nagao-Dias AT, Clinical Analysis and Toxicology Department, Faculty of Pharmacy, Federal University of Ceará, Rua Capitao Francisco Pedro, 1210,CEP 60430-370 Fortaleza, Ceará, Brazil, Phone: 55 - 85 3366-8270; Fax: 55 - 85 - 3366-8292; E-mail: tiemindi@yahoo.com.br

Received May 07, 2013; Accepted August 22, 2013; Published August 24, 2013

Citation: Teixeira FM, Vasconcelos LMF, Araújo TS, Genre J, et al. (2013) Association of CD69 and CD 25 Activation Markers on CD4 and CD8 Cells with Skin Tests in Drug Allergy. J Pharmacovigilance 1: 111. doi:10.4172/23296887.1000111

Copyright: (c 2013 Teixeira FM, et al. This is an open-access article distributed under the terms of the Creative Commons Attribution License, which permits unrestricted use, distribution, and reproduction in any medium, provided the original author and source are credited. 
Difficulties in DHR diagnosis also reside in variables such as the time period between drug exposure and reaction and the type of clinical manifestations observed in affected patients.

The lymphoproliferative test is widely used in diagnosis of immediate or non- immediate reactions. Nevertheless, it imposes technical problems, requires incorporation of radioactive substances and is time-consuming [9].

Because in many instances DHR affect the skin, the most common finding is composed mainly of activated $\mathrm{T}$ cells expressing activation markers such as CD69, IL-2R (CD25) and HLA-DR, and the skinhoming receptor cutaneous lymphocyte antigen in both CD4 and CD8 $\mathrm{T}$ cells with a predominance of one of them depending on the clinical manifestation $[5,10]$.

The aim of the present work was to search for CD25 and CD69 markers on T CD4+ and T CD8+ cells in drug allergy.

\section{Materials and Methods}

Fourteen patients with drug hypersensitivity attending the Dermatology Outpatient Clinic at the Hospital Universitário Walter Cantídio, Fortaleza, Brazil, were enrolled in this investigation. Some patients had at least one adverse reaction to one or more suspected drugs, therefore, a total of 16 reactions and 10 drugs were investigated. The reactions were considered to be non-immediate reactions once they occurred at least 2 hours after the drug administration. Immediate reactions occur within 1 hour after drug administration, and nonimmediate reactions occur later [10].

Healthy volunteers $(\mathrm{n}=6)$ without previous history of drug hypersensitivity were included in the study as controls for in vivo and in vitro drug testing.

Each of the participants of the study signed a written informed consent. The project, under code 011.03.08, was approved by the Ethics Committee in Research, Hospital Universitário Walter Cantídio, Federal University of Ceará, Brazil.

Drug skin testing included prick and patch tests and were applied according to standardized protocols established by the European Society of Contact Dermatitis Workshop [11].

Prick tests were performed on the forearm using the commercial form of the drugs. Reactions were considered positive when a wheal with a diameter $3 \mathrm{~mm}$ greater than that observed for the negative control ( $0.9 \%$ saline) was present 20 minutes after exposure to the drug.

Epicutaneous patch tests were performed on the patients' back using the commercial form of the drug or, whenever available, the pure substance. Tablets and capsule contents were evaluated at $10 \%$ and $30 \%$ in petrolatum. The results of patch testing were interpreted according to the International Contact Dermatitis Research Group [12] as negative, doubtful or positive after 48 hours, 72 hours and one week.

Peripheral blood mononuclear cells (PBMC) were cultured as described by Pichler and Tilch [13] with some modifications. Briefly, PBMC were obtained from heparinized peripheral blood by gradient centrifugation over Ficoll-Hypaque solution (SIGMA-ALDRICH, 3050 Spruce, St Louis, MO, USA). Concentrations of $1 \times 10^{6}$ cells $/ \mathrm{mL}$ in antibiotic-free RPMI 1640 (LGC BIOTECNOLOGIA, Rua Passadena, Sao Paulo, Brazil) supplemented with sodium bicarbonate $(2 \mathrm{~g} / \mathrm{L})$, l-glutamine $(2.05 \mathrm{mM})$ and $10 \%$ heat- inactivated AB-pooled serum, were cultured with various drug concentrations in 24-well roundbottom plates (TPP, CH-8219 Trasadingen, Schweiz, Switzerland) for 72 hours at $37^{\circ} \mathrm{C}$ and $5 \% \mathrm{CO} 2$ (tests were run in duplicate). PBMC incubated with $2.0 \mu \mathrm{g} / \mathrm{mL}$ phytohemagglutinin were considered as positive controls and those incubated with culture medium as negative controls. Pooled AB serum was obtained from healthy blood bank donors.

Before testing, drugs were dissolved in phosphate-buffered saline (PBS, LGC BIOTECNOLOGIA, Sao Paulo, Brazil) and adjusted to three different concentrations according to the procedures described by Martin et al. [7] and Pichler and Tilch [13]. Acetylsalicylic acid, diclofenac, rifamycin, phenytoin, paracetamol, sulfasalazine and betamethasone were previously dissolved in dimethyl sulphoxide, 0.1 M. The drugs were obtained from SIGMA-ALDRICH, St Louis, MO, USA, except for captopril (INCQS/FIOCRUZ, Av. Brasil, 4365, Rio de Janeiro Brazil) and etanercept (WYETH PHARMACEUTICALS Inc, Philadelphia, PA 19101, USA). The following concentrations were used: 1,10 and $100 \mu \mathrm{g} / \mathrm{mL}$ for paracetamol, dipyrone, diclofenac and betamethasone; 1,10 and $50 \mu \mathrm{g} / \mathrm{mL}$ for phenytoin, rifamycin and sulfasalazine. Acetylsalicylic acid was tested at 10,100 and $200 \mu \mathrm{g} / \mathrm{mL}$, and captopril at 1,10 and $100 \mu \mathrm{g} / \mathrm{mL}$. Etanercept was tested at 1,10 and $100 \mu \mathrm{g} / \mathrm{mL}$. Phytohemagglutinin (SIGMA-ALDRICH, St Louis, MO, USA) at $2.0 \mu \mathrm{g} / \mathrm{mL}$ was used as a positive control in the assays.

Expression of CD69 and CD25 on T CD4+ and CD8+ cells was assessed by flow cytometry (FACSCalibur, BD BIOSCIENCES, 2350 Qume Drive, San Jose, CA 95131, USA) with fluorochrome-labelled anti-human monoclonal antibodies PE-CD69, APC-CD25, FITC-CD4 and PerCP-CD8 (BD BIOSCIENCES, San Jose, CA, USA). The results were expressed as normalized mean fluorescence intensities (NMFI), which were calculated by multiplying the percentage of CD69+ or CD25+ positive cells by the mean fluorescence intensity of the markers, according to Beeler et al. [14]. Gating on lymphocytes was based on their forward and side scatter parameters, with an aquisition of 10,000 events colleted. Activation antigen expression was determined by setting quadrants using tube without antibodies and Fluorescence minus One (FMO). Analysis was performed using the FlowJo program. Stimulation indexes (SI) were calculated by the following formula: NMFI of the culture medium (CM) containing a certain drug concentration divided by NMFI of the CM without the drug. The result was considered to be positive when the SI was equal to or above 2.0, as proposed by Pichler and Tilch [13] and Beeler et al. [14].

Stimulation indexes of CD69 or CD25 in PBMC from drug allergic-patients were compared with those from controls by the MannWhitney non-parametric test for each drug concentration; a p-value less than 0.05 was considered statistically significant. The software used for statistical analysis was GraphPad Instat, version 3.10 (LaJolla, CA, USA).

\section{Results}

Each patient enrolled in the study presented a well-documented history of drug adverse reaction, symptoms of non-immediate reactions and a clear temporal correlation between drug exposure and symptoms. Drug allergy was classified according to the Naranjo adverse drug reactions probability scale [15]. Although NSAIDs are frequently related to non- allergic reactions, the possibility of a true IgE-mediated drug allergy cannot be excluded. For this reason, patients who presented clinical manifestations to NSAIDS were also included in the study. 
Citation: Teixeira FM, Vasconcelos LMF, Araújo TS, Genre J, et al. (2013) Association of CD69 and CD 25 Activation Markers on CD4 and CD8 Cells with Skin Tests in Drug Allergy. J Pharmacovigilance 1: 111. doi:10.4172/2329-6887.1000111

Page 3 of 6

Table 1: Patient's characteristics: Clinical manifestations, age of the patients, culprit drugs, positivity of skin tests and scores of the Naranjo adverse drug reactions probability scale.

\begin{tabular}{|c|c|c|c|c|c|c|c|}
\hline Id & $\begin{array}{c}\text { Age } \\
\text { (years) }\end{array}$ & Clinical manifestations & Culprit drug & Prick test & Patch test & Score Naranjo & Category \\
\hline & & Pruritus & ASA & Positive & n.d. & 6 & Probable \\
\hline 1 & 72 & Pruritus & DIC & Positive & n.d. & 6 & Probable \\
\hline 3 & 49 & Angioedema & ASA & Positive & n.d. & 10 & Definitive \\
\hline 4 & 61 & Angioedema & ASA & Positive & n.d. & 10 & Definitive \\
\hline 8 & 72 & Contact dermatitis & RIF & n.d. & Positive & 10 & Definitive \\
\hline 9 & 56 & Erytroderma & DIC & n.d. & Negative & 5 & Probable \\
\hline 10 & 65 & MPE & BET & n.d. & Negative & 6 & Probable \\
\hline 11 & 78 & Photoallergic reaction & ASA & n.d. & Positive & 7 & Probable \\
\hline 12 & 79 & Photoallergic reaction & CAP & n.d. & Positive & 7 & Probable \\
\hline 13 & 23 & FDE & PAR & n.d. & Negative & 6 & Probable \\
\hline 14 & 86 & DRESS & CAP & n.d. & Positive & 6 & Probable \\
\hline 15 & 53 & AGEP & ETN & n.d. & Negative & 7 & Probable \\
\hline 16 & 50 & Lichenoid eruption & DIP or DIC & n.d. & Negative & 6 & Probable \\
\hline 17 & 74 & Lichenoid eruption & CAP & n.d. & Positive & 6 & Probable \\
\hline 19 & 49 & SJS & SSZ & n.d. & Negative & 7 & Probable \\
\hline 20 & 64 & SJS & PHE & n.d. & Positive & 7 & Probable \\
\hline
\end{tabular}

Abbrevaitions: ASA: Acetylsalicylic acid, DIC: Diclofenac, DIP: Dipyrone, PAR: Paracetamol, CAP: Captopril, BET: Betamethasone, PHE: Phenytoin, RIF: Rifamycin, SSZ: Sulfasalazine, ETN: Etanercept, AGEP: Acute Generalized Exanthematous Pustulosis, DRESS: Drug Rash with Eosinophilia and Systemic Symptoms, FDE: Fixed Drug Eruption, SJS: Stevens-Johnson syndrome, MPE: Maculopapular exanthema, n.d.: not done..

CD4CD69

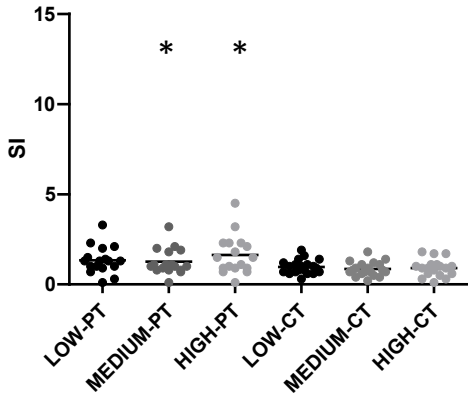

CD4CD25

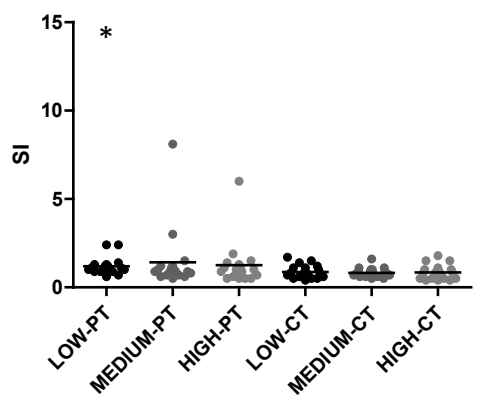

CD8CD69

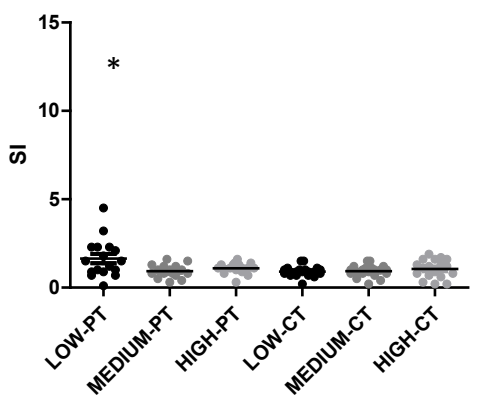

CD8CD25

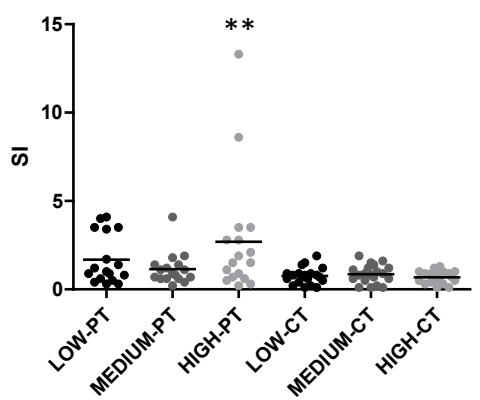

$* p \leq 0.05 ; * * p<0.01$ (Mann-Whitney test)

Figure 1: Stimulation index (Log2SI) for CD69 and CD25 markers on T CD4+ and T CD8+ lymphocytes in drug-allergic patients (PT) and in healthy individuals (CT) after 72 hours incubation with low, medium or high drug concentrations.

Table 1 presents data from the drug-allergic patients regarding clinical manifestations, culprit drugs, skin test, and the Naranjo Adverse Drug Reactions Probability. The prick test was positive in three cases due to acetylsalicylic acid and in one case due to diclofenac. The patch test was positive in one case of contact dermatitis caused by rifamycin and in one case of Stevens-Johnson syndrome caused by phenytoin.
Results were also positive in one case of DRESS, one case of lichenoid eruption and in two cases of photoallergic reaction due to captopril and ASA. The causality of drug adverse reaction was considered to be definitive in 3 cases and probable in 14 .

The period between drug allergy manifestations and in vitro testing varied from 1 to 41 months. Drug-allergic patients were evaluated 

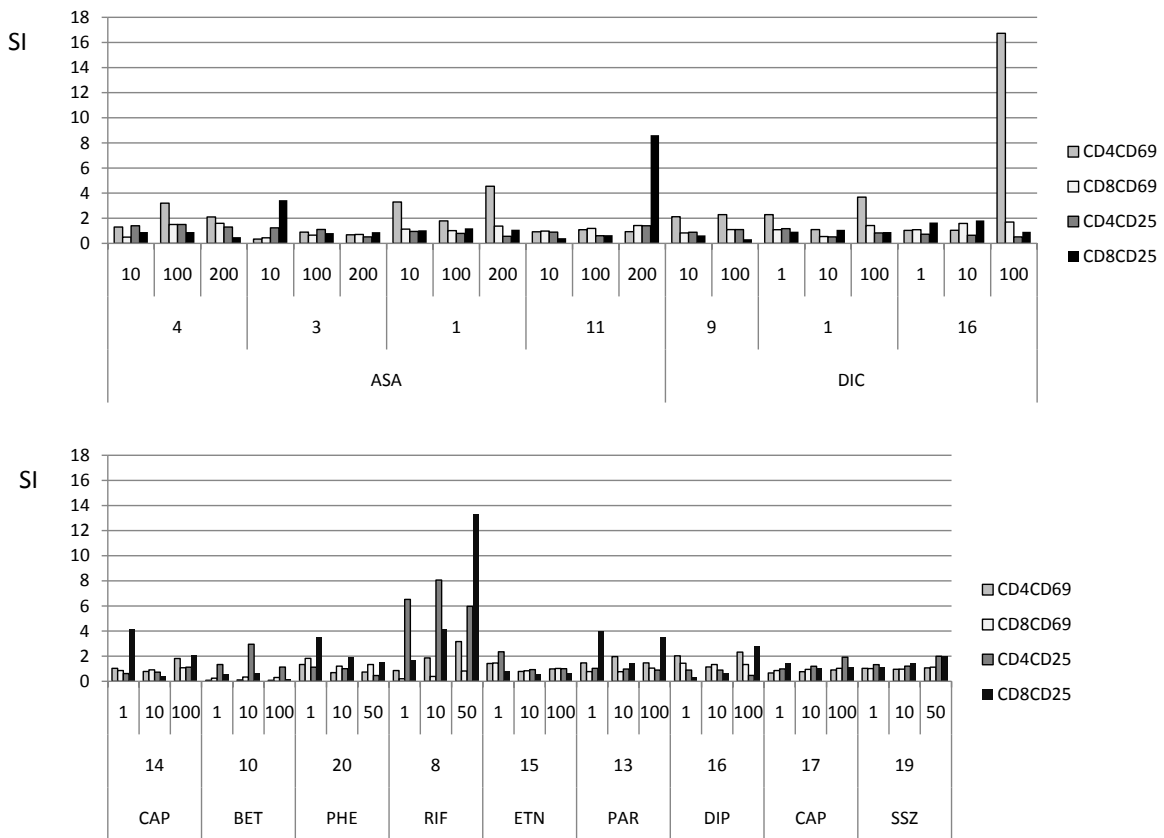

Figure 2: Drug-allergic patients presenting stimulation index (SI) of CD69 and CD25 on CD4+ and CD8+ T cells above 2 after incubation of the peripheral blood mononuclear cells with different concentrations $(\mu \mathrm{g} / \mathrm{mL})$ of the suspected drug.

for CD69 and CD25 upregulation on T CD4+ and T CD8+ cells after cell incubation with three different drug concentrations. First, the stimulation indexes of activation markers on CD4+ and CD8+ lymphocytes from drug- allergic patients (PT) were compared with those from healthy individuals (CT), irrespective of the suspected drug (Figure 1). Statistical differences were found at medium and high drug concentrations for the CD4+CD69+ marker $(p \leq 0.05)$, at the lowest drug concentration for the CD4+CD25+ and CD8+CD69+ markers $(p \leq 0.05)$ and at the highest drug concentration for the CD8 + CD25 + marker $(p<0.01)$, when samples from patients were compared to controls.

One or both the markers were upregulated in 3 patients who had positive results in prick test. Four out of six patients who presented positive patch test showed upregulation of one or both the activation markers. An upregulation of CD25 on CD8+ T cells (Figure 2) was found in one case of DRESS (patient \#14), Steven-Johnson syndrome (\#20), fixed drug eruption (\#13) an in one case of photoallergic contact dermatitis (\#12). The patient \#1 who suffered from pruritus after the diclofenac and ASA ingestion showed a positive prick test for both drugs and presented upregulation of CD69 on CD4+ cells. The patient \#8 showed upregulation of CD69 on CD4+ cells, and CD25 on CD4+ and CD8+ cells. He suffered from contact dermatitis to rifamycin and presented positive patch test to the culprit drug. The 200 patient \#16 suffered from liquenoid eruption, presented negative patch test results but showed upregulation of CD69 on CD4+ cells when PBMC were tested with diclofenac and upregulation of both CD69 on CD4+ cells and CD25 on CD8+ cells when PBMC were tested with dipyrone.

\section{Discussion}

In vivo tests as the epicutaneous tests are used as the first line of investigation in non-immediate reactions, including fixed drug eruption, contact dermatitis, erythroderma (exfoliative dermatitis), maculopapular exanthema, lichenoid eruption, acute generalized exanthematous pustulosis and drug rash with eosinophilia and systemic symptoms. Some researchers have suggested that intradermal tests with immediate and delayed readings should be included in the investigation of delayed hypersensitivity to drugs [16]. Considering our patients, the intradermal tests might be useful in cases when the patch tests were negative, as in erythroderma, lichenoid and maculopapular drug eruptions.

In the case of drug fixed eruption, it is often suggested that the patch test be performed on the residual pigmented skin site of the reaction [16]. In our study, the test was performed on the patient's back and the result was negative. The patient did not permit the test to be performed at the same place of the reaction.

In cases of SJS, the IDT is not recommended due to the possibility of reproducing the reactions. The patch test has a weak sensitivity in those circumstances and few cases of positive results have been reported. Wolkenstein et al. [17] found 2 out of 22 cases of SJS/TEN with positive results. Others have reported positivity for carbamazepine [18] and phenytoin [19]. In our study, patch test was positive in a patient who presented SJS due to phenytoin but was negative in a patient who suffered from SJS caused by sulfasalazine.

Although it is expected a good sensitivity of patch test in AGEP [16], the result was negative. At the time of the testing, the patient was using another anti-TNF drug what could partially explain the negative results. According to Wee et al. [20], a significant positivity of patch tests can be found even in patients under use of systemic corticosteroids and cytokine inhibitors such as adalimumab, etanercept and infliximab. In respect to the subject of 12 performing patch test with anti-TNF, it is accepted by some authors [21] and questioned by others [22]. The major concern is related to the fact that this type of biological agent has high molecular weight $(>500 \mathrm{Da})$, which could reduce its penetration of skin. The results of in vivo tests can vary substantially depending on the 
Citation: Teixeira FM, Vasconcelos LMF, Araújo TS, Genre J, et al. (2013) Association of CD69 and CD 25 Activation Markers on CD4 and CD8 Cells with Skin Tests in Drug Allergy. J Pharmacovigilance 1: 111. doi:10.4172/2329-6887.1000111

Page 5 of 6

period of drug sensitization, which may decrease over time [16]. That means that negative results do not exclude drug allergy.

In vitro tests are not commonly performed during the acute phase of the reactions because the cells are strongly activated and can result in high background proliferation. In general, the shortest interval recommended for doing in vitro tests is at least three weeks after the clinical manifestations [5]. For lymphoproliferative assays, Pichler and Tilch [13] observed that the test could be performed 10 to 20 years after the drug reaction. CD69 upregulation on CD4+ and CD8+ T cells in patients at intervals of 2 months to 12 years after the adverse drug reactions has been demonstrated [14]. In our study, we have analyzed patients who had presented the clinical manifestations 1 to 41 months before testing.

Different activation markers such as CD25 (IL-2R), CD69, CD71 and HLA-DR are known to be expressed on the surface of T cells after stimulation $[5,10]$.

Beeler et al. [14] demonstrated the usefulness of identifying CD69 upregulation on T cells in maculopapular exanthema, Steven Johnson syndrome, DRESS, AGEP. We also found its upregulation in one case of contact dermatitis, in one case of FDE, in two cases of pruritus, one case of angioedema, two cases of liquenoid eruption, and in one case of erythroderma.

Miki-Hosokawa et al. [23], using a mouse model of allergic asthma, demonstrated that antigen-induced airway inflammation and hyperresponsiveness were highly associated to CD69-dependent migration of Th2 cells into the asthmatic lung. The authors mention that CD69 was also associated with Th1-induced airway inflammation.

Longo et al. [24] observed that CD69, CD25 and HLA-DR expressions were increased in two patients who had presented IgEmediated allergy to clavulanic acid but had showed negative skin test results. They found an increase in the expression of CD69 within 4 hours of culture and that CD25 and HLA-DR were upregulated within 24-48 hours of culture. Torres et al. [10] had observed an increase of CD69 after the 3rd day of the reaction on both TCD4 and TCD8 positive cells, and a significant increase of CD25, cutaneous-lymphocyte antigen and HLA-DR after the 15th day of the reaction on CD4 cells in skin biopsies from patients with allergy to iodine contrast media. The values were unchanged in CD8 cells.

Nishio et al. [25] observed that no CD69 was expressed on ex-vivo peripheral blood mononuclear cells (onset to recovery) in patients with maculopapular exanthema, SJS and in drug-induced hypersensitivity syndrome. The CD25 marker, instead, was increased on both CD4+ and CD8+ T cells. They also demonstrated that these cells were activated once they did not present CTLA- 4 .

We have found upregulation of CD8+CD25+ T cells in 7 out of 17 samples (41.1\%) and of CD4+CD25+ T cells in 2 out of 7 samples (11.7\%). We did not evaluate if CD4+CD25+ and CD8+CD25+ cells were regulatory $\mathrm{T}$ cells. The regulatory cell population is determined by the expression of CTLA-4 and X linked forkhead/winged helix transcription factor (FoxP3) [26]. CD8+ T cells may also participate in regulatory function. They may active regulate inflammation and they differ from the citotoxic population because they do not express CD28 and may express CD38. Eusebio et al. [27] had observed higher frequencies CD8+CD25+FoxP3- T cells in patients with asthma and that they were correlated with asthma severity.

Finally, our data reinforce the use of CD69 and CD25 on both CD4+ and CD8+ T cells in order to investigate drug allergy.

\section{Acknowledgement}

This study was financially supported by the CNPq (process 554970/2010-4) and FUNCAP/CAPES (process BMD - 1401-2.08/08.

\section{References}

1. Lochmatter P, Zawodniak A, Pichler WJ (2009) In vitro tests in drug hypersensitivity diagnosis. Immunol Allergy Clin North Am 29: 537-554.

2. Demoly P, Viola M, Gomes ER, Romano A. (2007). Epidemiology and causes of drug hypersensitivity. In: Pichler, WJ, ed. Drug Hypersensitivity. Basel: Karger, 2-17.

3. Gomes ER, Demoly P (2005) Epidemiology of hypersensitivity drug reactions Curr Opin Allergy Clin Immunol 5: 309-316.

4. Thong BY, Tan TC (2011) Epidemiology and risk factors for drug allergy. $\mathrm{Br} \mathrm{J}$ Clin Pharmacol 71: 684-700.

5. Porebski G, Gschwend-Zawodniak A, Pichler WJ (2011) In vitro diagnosis of $T$ cell-mediated drug allergy. Clin Exp Allergy 41: 461-470.

6. Khan DA, Solensky R (2010) Drug allergy. J Allergy Clin Immunol 125: S126137.

7. Martin M, Wurpts G, Ott H, Baron JM, Erdmann S, et al. (2010) In vitro detection and characterization of drug hypersensitivity using flow cytometry. Allergy 65 32-39.

8. Torres MJ, Mayorga C, Blanca M (2009) Nonimmediate allergic reactions induced by drugs: pathogenesis and diagnostic tests. J Investig Allergol Clin Immunol 19: 80-90.

9. Nyfeler B, Pichler WJ (1997) The lymphocyte transformation test for the diagnosis of drug allergy: sensitivity and specificity. Clin Exp Allergy 27: 175181.

10. Torres MJ, Mayorga C, Cornejo-Garcia JA, Lopez S, Chaves P, et al. (2008) Monitoring non-immediate allergic reactions to iodine contrast media. Clin Exp Immunol 152: 233-238.

11. Barbaud A, Gonçalo M, Bruynzeel D, Bircher A; European Society of Contact Dermatitis (2001) Guidelines for performing skin tests with drugs in the investigation of cutaneous adverse drug reactions. Contact Dermatitis 45: 321 328 .

12. Lachapelle J-M, Maibach HI. (2009).Patch testing and prick testing: A practical guide. 2nd ed. Verlag-Berlin Heidelberg, 203p.

13. Pichler WJ, Tilch J (2004) The lymphocyte transformation test in the diagnosis of drug hypersensitivity. Allergy 59: 809-820.

14. Beeler A, Zaccaria L, Kawabata T, Gerber BO, Pichler WJ (2008) CD69 upregulation on $\mathrm{T}$ cells as an in vitro marker for delayed-type drug hypersensitivity. Allergy 63: 181-188.

15. Naranjo CA, Busto U, Sellers EM, Sandor P, Ruiz I, et al. (1981) A method for estimating the probability of adverse drug reactions. Clin Pharmacol Ther 30 : 239-245.

16. Barbaud A (2009) Skin testing in delayed reactions to drugs. Immunol Allergy Clin North Am 29: 517-535.

17. Wolkenstein P, Chosidow O, Fléchet ML, Robbiola O, Paul M, et al. (1996) Patch testing in severe cutaneous adverse drug reactions, including StevensJohnson syndrome and toxic epidermal necrolysis. Contact Dermatitis 35: 234 236 .

18. Lin YT, Chang YC, Hui RC, Yang CH, Ho HC, et al. (2013) A patch testing and cross-sensitivity study of carbamazepine-induced severe cutaneous adverse drug reactions. J Eur Acad Dermatol Venereol 27: 356-364

19. Sharma VK, Vatve M, Sawhney IM, Kumar B (1998) Clinical spectrum of drug rashes due to antiepileptics. J Assoc Physicians India 46: 595-597.

20. Wee JS, White JM, McFadden JP, White IR (2010) Patch testing in patients treated with systemic immunosuppression and cytokine inhibitors. Contact Dermatitis 62: 165-169.

21. Seneschal J, Lepreux S, Milpied B, Schaeverbeke T, Taïeb A (2007) Psoriasiform eruptions during anti TNF-alpha treatment: psoriasis or not? Arch Dermatol 143: 1593-1595.

22. Lecluse LL, Piskin G, Bos JD (2008) The use of patch tests in determining hypersensitivity to etanercept and infliximab. Arch Dermatol 144: 1070-1071. 